\title{
Commémorer la ville : une analyse comparative des célébrations du centenaire de Toronto et du tricentenaire de Montréal
}

\section{Harold Bérubé}

Volume 57, numéro 2, automne 2003

URI : https://id.erudit.org/iderudit/009143ar

DOI : https://doi.org/10.7202/009143ar

Aller au sommaire du numéro

Éditeur(s)

Institut d'histoire de l'Amérique française

ISSN

0035-2357 (imprimé)

1492-1383 (numérique)

Découvrir la revue

Citer cet article

Bérubé, H. (2003). Commémorer la ville : une analyse comparative des célébrations du centenaire de Toronto et du tricentenaire de Montréal. Revue d'histoire de l'Amérique française, 57(2), 209-236.

https://doi.org/10.7202/009143ar

\section{Résumé de l'article}

Cet article constitue une étude comparative des célébrations commémoratives entourant le centenaire de l'incorporation de Toronto en 1934 et le tricentenaire de la fondation de Montréal en 1942. Ces deux événements sont l'occasion de s'exprimer sur le milieu urbain, sur sa relation avec l'ensemble plus large qu'est la nation et, plus généralement, avec l'Occident. Au-delà des particularités des contextes torontois et montréalais, une approche commune de la commémoration et des discours et des pratiques qui y sont associées se dégage. Toutefois, comme le démontre une analyse du personnel engagé dans l'organisation de ces fêtes, des programmes qu'ils ont élaborés et des résultats finaux, ces discours et ces pratiques commémoratives sont aussi modulés par des impératifs de nature plus pragmatique qu’idéologique, plus matérielle que culturelle. 


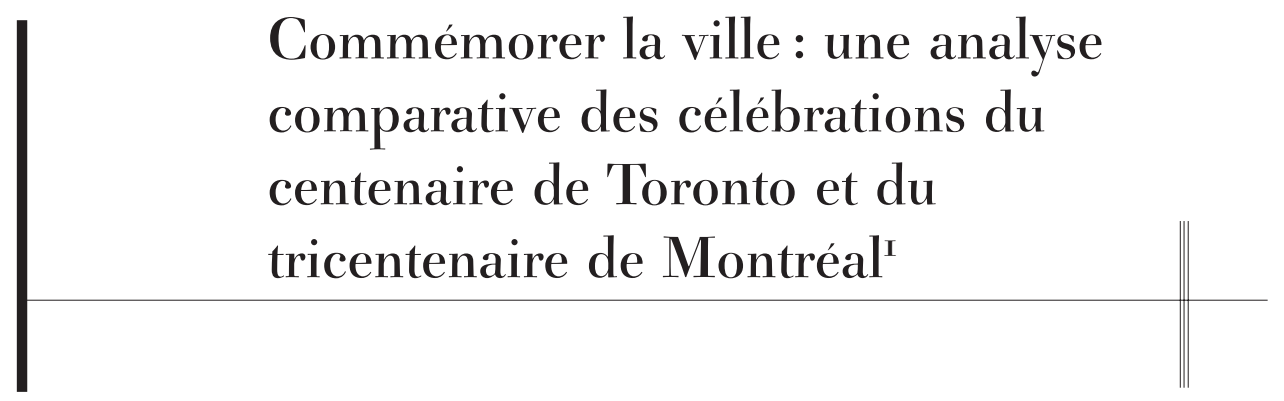

HAROLD BÉRUBÉ

Département d'histoire

Université du Québec à Montréal

RÉSUMÉ - Cet article constitue une étude comparative des célébrations commémoratives entourant le centenaire de l'incorporation de Toronto en 1934 et le tricentenaire de la fondation de Montréal en 1942. Ces deux événements sont l'occasion de s'exprimer sur le milieu urbain, sur sa relation avec l'ensemble plus large qu'est la nation et, plus généralement, avec l'Occident. Au-delà des particularités des contextes torontois et montréalais, une approche commune de la commémoration et des discours et des pratiques qui y sont associées se dégage. Toutefois, comme le démontre une analyse du personnel engagé dans l'organisation de ces fêtes, des programmes qu'ils ont élaborés et des résultats finaux, ces discours et ces pratiques commémoratives sont aussi modulés par des impératifs de nature plus pragmatique qu'idéologique, plus matérielle que culturelle.

ABSTRACT - This article is a comparative study of the commemorative celebrations of the $100^{\text {th }}$ anniversary of Toronto's incorporation in 1934 and the $300^{\text {th }}$ anniversary of the foundation of Montréal by de Maisonneuve in 1942. Both events make place to discourses on the urban environment, its relation with the larger ensemble that is the nation and,

1. Cet article est tiré de mon mémoire de maîtrise (Harold Bérubé, Commémorer la ville, une analyse comparative des célébrations du centenaire de Toronto en 1934 et du tricentenaire de Montréal en 1942, mémoire de maîtrise (Histoire), Université de Montréal, 2002, 166 p.). Sa rédaction n’aurait pas été possible sans l'aide financière du CRSH et du FQRSC. Je souhaite d'ailleurs remercier mes correcteurs anonymes dont les commentaires et critiques ont grandement contribué à la forme finale de cet article, ainsi qu'Amélie Bourbeau, Jean-Pierre Collin et Michèle Dagenais, qui en ont lu les premières versions. 
generally, with the Western civilization. Beyond the specific contexts of Toronto and Montréal, a common approach to commemoration, discourses and the associated practices can be drawn. However, as demonstrated by an analysis of the actors involved in the organization of these festivities and of the programs they prepared, the end result of their efforts is as much a product of ideological and cultural imperatives as of material and pragmatic concerns.

$\mathrm{Q}$ UE FAISONS-NOUS lorsque nous commémorons? S’agit-il d'évoquer et d'honorer le passé, de répondre mécaniquement aux appels du calendrier et de l'histoire, ou n'est-ce encore qu'un prétexte pour se divertir? La commémoration est-elle un outil au service d'élites désirant manipuler la mémoire collective à leurs fins ou plus simplement une entreprise commerciale et touristique? L'acte de commémoration est complexe. Il tend vers plusieurs fins contradictoires et répond simultanément à plusieurs besoins.

Ce n’est que depuis les années 1980 que les célébrations commémoratives et autres manifestations spectaculaires de l'identité collective font l'objet d'études historiques approfondies ${ }^{2}$. Les deux dernières décennies ont ainsi vu se multiplier les travaux portant sur les traditions inventées, les lieux de mémoire et autres avatars de la mémoire publique ${ }^{3}$. Comme la majorité des auteurs de ces ouvrages, je crois que ce type de célébration est plus qu'une simple affaire de divertissement. La commémoration est une occasion pour les élites d'une société d'utiliser l'histoire, la symbolique, les discours et les actes en vue de modifier ou de renforcer un

2. Cette question est explorée dans le bref bilan dressé par Nicholas Rogers et Adrian Shubert, "Introduction: Spectacle, Monument, and Memory", Histoire sociale/Social History, 29,58 (novembre 1996): 265-273.

3. Mentionnons les ouvrages clés d'Eric Hobsbawm et Terence Ranger, dir., The Invention of Tradition (Cambridge, Cambridge University Press, 1999; 1983), 322 p.; et de Pierre Nora, dir. Les lieux de mémoire (Paris, Gallimard, 1984), 3 vol. Plus près de nous, en plus d'une pléiade d'articles, mentionnons, malgré leurs limites, les ouvrages de Jacques Lacoursière et Jacques Mathieu, Les mémoires québécoises (Sainte-Foy, Les Presses de l'Université Laval, 1991), 383 p.; et de Rémi Tourangeau, Fêtes et Spectacles du Québec (Québec, Nuit Blanche, 1992), 398 p. On retrouvera des études plus fouillées dans Norman Knowles, Inventing the Loyalists. The Ontario Loyalist Tradition \& the Creation of Usable Pasts (Toronto, University of Toronto Press, 1997), 244 p. ; Jonathan F. Vance, Death So Noble: Memory, Meaning and World War One (Vancouver, UBC Press, 1997), 319 p. ; Patrice Groulx, Pièges de la mémoire: Dollard des Ormeaux, les Amérindiens et nous (Hull, Vents d'Ouest, 1998), 436 p.; Henry Vivian Nelles, The Art of Nation-Building. Pageantry and Spectacle at Quebec's Tercentenary (Toronto, University of Toronto Press, 1999), 397 p. ; Alan Gordon, Making Public Pasts. The Contested Terrain of Montreal's Public memories, 1891 1930 (Montréal, McGill-Queen's University Press, 2001), 233 p.; et, plus récemment, Ronald Rudin, Founding Fathers. The Celebration of Champlain and Laval in the Streets of Quebec, 1878-1908 (Toronto, University of Toronto Press, 2003), 290 p. 
ou des cadres identitaires ${ }^{4}$. Si ce processus est inhérent aux célébrations commémoratives, il est limité par un ensemble de contraintes d'ordre idéologique et culturel, mais aussi d'ordre matériel et pragmatique. Ces derniers éléments ont d'ailleurs souvent peu à voir avec la construction de l'identité publique ou collective. Il n'en demeure pas moins qu'ils ont parfois une influence déterminante sur le déroulement des festivités en question. C'est une problématique qu'illustrent d'ailleurs pleinement les deux séries de célébrations commémoratives étudiées ici: celles du centenaire de l'incorporation de Toronto en 1934 et celles du tricentenaire de la fondation de Montréal en 1942.

Cette étude veut mettre en évidence le double rôle joué par ces contraintes culturelles et matérielles dans l'organisation et le déroulement de ces fêtes. Dans un premier temps, j'évoquerai brièvement le contexte immédiat des fêtes, c'est-à-dire les conditions de préparation et de déroulement des festivités; deuxièmement, je m'attarderai au contexte historique plus large des deux villes concernées. Cette première démarche permettra de mieux comprendre la sélection des événements historiques qui, dans chacune des deux villes, font l'objet d'une commémoration.. Ce décor mis en place, j'aborderai les festivités en tant que telles. J'évoquerai les individus et les groupes qui s'y sont engagés, les programmes élaborés. Cette seconde démarche éclairera les changements qui s'opèrent entre la conception initiale des programmes et le déroulement effectif des festivités.

Un mot sur les sources utilisées dans le cadre de cette étude. Les deux principaux fonds dépouillés sont ceux des deux comités organisateurs 5 . Dans les deux cas, il existe également des publications qui commémorent les fêtes. Dans le cas de Toronto, une monographie sur l'histoire de la ville de l'historien J. E. Middleton ${ }^{6}$ a été publiée. Du côté de Montréal, le secrétaire du comité des fêtes, Jean-Paul Héroux, a produit un compte

4. Cet aspect - l'utilisation de l'histoire à des fins identitaires - a déjà été abordé dans Harold Bérubé, "La ville au cœur de la nation: l'utilisation du passé dans l'élaboration de l’identité urbaine ", Urban History Review/Revue d'histoire urbaine, 30,2 (mars 2002): 16-27.

5. Montréal, Division de la gestion des documents et des archives (DGDA), fonds de la Commission du Troisième Centenaire de Montréal (VM 12); Toronto, City of Toronto Archives (CTA), fonds du Toronto Centennial Celebrations Committee (RG 250). Soulignons que dans le cas torontois, il existe également un fonds provincial lié aux fêtes: Toronto, Archives of Ontario, Toronto Centennial collection (MU 2989-2990). Malheureusement, ce fonds ne contient que des documents promotionnels ou officiels déjà accessibles par l'entremise du fonds municipal.

6. J. E. Middleton, The Officiel Centennial Book 1834-1934. Toronto's 100 Years (Toronto, The Centennial Committee, 1934), $228 \mathrm{p}$. 
rendu très détaillé des fêtes ${ }^{7}$. L’enthousiasme de Héroux appelle toutefois à la prudence quant à son utilisation pour autre chose que des données factuelles. Enfin, pour les deux villes, j’ai procédé à un dépouillement des grands quotidiens locaux pour la durée des fêtes et pour les moments décisifs de leur organisation ${ }^{8}$.

Le principal objectif de cet article est de relativiser l'importance attribuée aux impératifs d'ordre culturel ou idéologique dans la formation des discours et des pratiques identitaires associés à ce genre de manifestation. Sans les réduire à un bruit de fond insignifiant, je désire souligner que, s'ils nous renseignent sur la mentalité des élites commémorantes qui ont élaboré ces fêtes, ces discours et ces pratiques sont également modulés par des considérations d'ordre pragmatique ou matériel. On verra que les contextes difficiles dans lesquels se déroulent ces fêtes viennent accentuer ce dernier aspect.

\section{LE CONTEXTE HISTORIQUE}

Les réalités économiques, politiques et sociales auxquelles doivent faire face les commémorants, si elles n'expliquent pas à elles seules la tenue de célébrations commémoratives, en déterminent l'ampleur, le caractère et, dans une moindre mesure, le contenu. C'est donc vers ces éléments de contexte que je me tournerai d'abord. À Toronto comme à Montréal, les organisateurs des fêtes étudiées devront composer avec des contraintes importantes, mais de différentes natures. Ainsi, les cérémonies du centenaire de l'incorporation de Toronto seront préparées et se dérouleront dans l'ombre de la crise économique qui balaie la majeure partie du monde occidental tout au long de la décennie 1930. Les fêtes du tricentenaire de Montréal se tiendront dans le sillage de cette crise, mais dans le contexte encore plus troublant d'une guerre mondiale.

Sans être totalement épargnée par la Crise, Toronto s'illustrera tout au long de la décennie 1930 par sa capacité à résister à ses pires effets. À l'opposé, Montréal les subira avec difficulté. Dans les deux régions métropolitaines, un nombre important de municipalités périphériques font face à la banqueroute. Montréal elle-même n'échappe pas à cette situation. Au contraire, Toronto parvient à gérer ses déficits successifs et

7. Jean-Paul Héroux, 1642-1942: Troisième Centenaire de Montréal (Montréal, Commission du IIIe centenaire, 1942), $302 \mathrm{p}$.

8. Il s'agit des quotidiens le Globe et le Daily Star pour Toronto, et de La Patrie, La Presse et The Gazette pour Montréal. 
à maintenir son taux de chômage à des niveaux raisonnables ${ }^{9}$. Ce contraste entre les deux villes se retrouve aussi du point de vue de leurs destinées financières. La métropole ontarienne conserve ses acquis ${ }^{10}$. À l'opposé, la situation économique de sa concurrente québécoise se détériore rapidement dès les premières années de la Crise. Certains de ses piliers financiers, comme la Sun Life ou la Banque de Montréal, s'en tirent assez bien, mais sa Bourse connaît un interminable purgatoire dont les effets se feront sentir jusque dans les années d'après-guerre.

À ce niveau, on peut dire que la Crise ne fait qu'accentuer des tendances lourdes déjà présentes depuis au moins la fin de la Première Guerre mondiale. Le déclin progressif de la Grande-Bretagne comme puissance économique et principal partenaire commercial des élites économiques de Montréal, conjugué à une ascension de l'économie américaine pour laquelle Toronto est mieux préparée et située, condamnent à long terme la prédominance de l'économie montréalaise au Canada et favorisent la montée de sa rivale ontarienne ${ }^{11}$. Cette double trajectoire se reflétera dans les discours et les pratiques observés dans les deux villes. Plus largement, le contexte de crise économique est inhérent à l'évolution générale de l'opinion publique et des mentalités durant cette période. Même si ces données ne peuvent être chiffrées, elles entrent indéniablement dans les calculs des organisateurs des deux séries de fêtes étudiées ici.

À travers le Canada, on retrouve les mêmes symptômes à des degrés divers: effritement de la confiance de la population envers les institutions politiques et économiques traditionnelles, montée de l'agitation et de la radicalisation politiques, xénophobie latente. À Toronto comme à Montréal, ces effets sont modulés par les particularités du contexte local. Dans le premier cas, la relative aisance avec laquelle la ville traverse la crise, la présence au niveau municipal d'un gouvernement conservateur bénéficiant d'un support important et l'action ponctuelle des forces policières contribuent à freiner l'éclosion de mouvements radicaux ou le

9. Roger E. Riendeau, "A Clash of Interests: Dependency and the Municipal Problem in the Great Depression", Revue d'études canadiennes, 14,1 (printemps 1979): 50.

10. Par exemple, le Financial Post est en mesure, en 1939, de proclamer que Toronto "[has] passed Montreal as a centre of finance, commerce and industry. "; cité dans James Lemon, Toronto Since 1918: An Illustrated History (Toronto, J. Lorimer, 1985), 64.

11. Paul-André Linteau, Histoire de Montréal depuis la Confédération (Montréal, Boréal, 2000), 286, 305-306. 
désordre social ${ }^{12}$. Ce qui n'empêche pas différents courants progressistes plus modérés et issus des milieux ouvriers d'avoir un impact sur le plan idéologique. On en retrouvera par exemple la trace dans un vibrant plaidoyer du lieutenant-gouverneur ontarien $\mathrm{H}$. A. Bruce en faveur du logement social lors d'une des cérémonies d'ouverture du Centenaire ${ }^{13}$. On notera tout de même au sein des membres de l'élite locale engagés dans l'organisation des fêtes du centenaire un attachement aux institutions et aux valeurs de l'empire. L'importante homogénéité culturelle et ethnique de la métropole ontarienne, ainsi que la place considérable qu'y occupent traditionnellement l'imagerie et les valeurs impériales, favorisent cette tendance qui ne doit toutefois pas être associée à un impérialisme traditionaliste. L'attachement dont il est question n'a plus beaucoup à voir avec l'impérialisme victorien décrit par Carl Berger pour la période $1867-1914^{14}$. On ne rêve plus tant à un empire confédéral fort qu'à une relation surtout culturelle et politique entre partenaires égaux.

Plus concrètement, en 1934, le conservatisme ambiant dont il est question trouve un écho dans la structure politique du pays : à Ottawa, R. B. Bennett et les Conservateurs sont en selle depuis 1930; en Ontario, George S. Henry a remplacé le "grand prêtre tory» G. Howard Ferguson à la tête du parti conservateur provincial; à Toronto, le conservateur William Stewart a accédé en 1930 à la mairie. La fragilité de cette emprise conservatrice sur les structures politiques ontariennes est toutefois mise en évidence par le fait que l'année même des fêtes du centenaire, Henry et Stewart quittent le pouvoir. Battu par King, Bennett les suivra l'année suivante.

Pour ce qui est de Montréal, au niveau économique, la Seconde Guerre mondiale se traduit rapidement par une certaine prospérité, même si les séquelles de la crise continuent de se faire sentir ${ }^{15}$ et même si la guerre entraîne son lot d'incertitudes. Ajoutons à ce contexte les

12. "While the dependency problem tended to make municipal politics more lively in the 1930s, it did not precipitate widespread radicalism and political upheaval in the metropolitan Toronto area. There is no significant shift to the "left"...», R. E. Riendeau, loc. cit., 56.

13. Le lieutenant-gouverneur attaque franchement les autorités municipale, provinciale et fédérale pour leur passivité face à la dégradation rapide de la situation du logement dans la VilleReine, qui se veut pourtant exemplaire au Canada à ce sujet. City of Toronto Archives (CTA), Toronto City Council Minutes, 1934, appendice C, 24-26.

14. Carl Berger, The Sense of Power. Studies in the Ideas of Canadian Imperialism 1867-1914 (Toronto, University of Toronto Press, 1970), 3-11.

15. La mise en tutelle de la ville de Montréal de 1940 à 1944 , pour cause de surendettement et de mauvaise administration, constitue une manifestation particulièrement spectaculaire de ces effets. Paul-André Linteau et al., Histoire du Québec contemporain. Le Québec depuis 1930, tome II (Montréal, Boréal, 1989), 63. 
débats et le plébiscite de 1942 sur la conscription, qui ont lieu parallèlement aux fêtes du tricentenaire et qui réveillent à travers le Canada le spectre d'une crise semblable à celle qu'avait connue le pays durant le conflit précédent. Enfin, dans le cadre d'un réveil du nationalisme canadien-français, qui se fait clairement sentir dès les années 1920 et 1930, Montréal devient rapidement un «champ de bataille» symboliquement significatif. Tout au long de l'entre-deux-guerres, le poids démographique des francophones augmente dans une ville où les anglophones exercent toujours une importante influence économique, politique et culturelle. La volonté de reconquérir la ville, de se l'approprier politiquement et symboliquement se fera sentir lors des fêtes ${ }^{16}$.

Dans ce contexte, on constatera que les festivités étudiées seront l'occasion de véhiculer des valeurs et des messages émanant principalement de groupes et d'individus liés aux composantes plus conservatrices de leurs sociétés respectives. Il s'agit autant pour ces acteurs de freiner ou de contrer les changements liés à la modernité que connaissent leurs sociétés respectives, que de trouver les moyens de s'y adapter. Malgré ces efforts, il est important de noter que ces élites conservatrices - les tenants du clérico-nationalisme dans un cas, ceux d'un nationalisme canadien teinté d'impérialisme dans l'autre - verront leurs bases sociales et les valeurs sur lesquelles elles s'appuient s'effriter rapidement dans les années d'après-guerre. Les axes identitaires autour desquels gravitent les deux Canadas changeront après la Deuxième Guerre mondiale, reléguant les spectacles, les rituels et les discours décrits plus bas aux oubliettes de la mémoire publique ${ }^{17}$, et rendant inutilisables à des fins identitaires certains aspects du passé mis de l'avant lors des fêtes.

Car, à la base, à Montréal comme à Toronto, un moment premier a été favorisé par les commémorants aux dépens d'autres possibilités ${ }^{18}$, les

16. La question du déclin de la présence des anglophones dans la «mémoire publique» de la ville a été brièvement explorée par Alan Gordon, Making Public Pasts. The Contested Terrain of Montreal's Public Memories, 1891-1930 (Montréal, McGill-Queen's University Press, 2001), 178-183.

17. Les changements identitaires importants que connaissent le Canada anglais et le Canada français au cours des décennies qui suivent la fin de la Deuxième Guerre mondiale sont décrits, entre autres, dans Kenneth McRoberts, Misconceiving Canada. The Struggle for National Unity (Oxford, Oxford University Press, 1997), 31-76; José E. Igartua, «L'autre révolution tranquille. L'évolution des représentations de l'identité canadienne-anglaise depuis la Deuxième Guerre mondiale», dans Gérard Bouchard et Yvan Lamonde, dir., La nation dans tous ses états. Le Québec en comparaison (Montréal, Harmattan, 1997), 271-296; Susan Mann, The Dream of Nation. A Social and Intellectual History of Quebec (Montréal, McGill-Queen’s University Press, 2002) (1982), 266-315.

18. "Montréal, like many cities established in the New World, has numerous "foundations". ", A. Gordon, op. cit., 175. 
plus évidentes étant la fondation de Toronto/York par le lieutenantgouverneur du Haut-Canada, John Graves Simcoe, en 1793 et, inversement, l'obtention par Montréal en 1833 d'une charte municipale. Les événements choisis et surtout la manière dont ils sont présentés ou mis en marché reflètent non seulement une certaine historiographie, mais surtout une vision du passé guidée par le présent.

Dans le cas de Montréal, il existait, longtemps avant la professionnalisation de la discipline historique au Canada, tout un corpus sur les origines de Ville-Marie ${ }^{19}$. Des auteurs comme Dollier de Casson et ÉtienneMichel Faillon posent les bases d'un récit de fondation de Ville-Marie où se mêlent héroïsme, mystique missionnaire et intervention divine. Paul Chomedey de Maisonneuve, fondateur de la ville, y prend la tête d'un groupe de personnages plus grands que nature. Guidés par la Providence en terre d'Amérique, ils parviendront, malgré les obstacles que sont les Sauvages et la nature inhospitalière, à poser les bases d'une grande ville. C'est essentiellement à ce récit teinté de légendaire que se référeront les organisateurs des cérémonies commémoratives ${ }^{20}$. Comment se mesure-t-il à l'histoire de Montréal écrite de nos jours?

Le site de Montréal attire l'attention de Samuel de Champlain plusieurs années avant la fondation d'un établissement européen permanent sur l'île. Il faut attendre 1642 pour que, sous la gouverne de Paul Chomedey de Maisonneuve, un groupe d'une quarantaine de colons y débarque et y prenne résidence. Ville-Marie missionnaire vivote durant ses premières années d'existence et ne prend son envol qu'en s'insérant dans le réseau de commerce de la fourrure dont dépend en bonne partie l'économie de la Nouvelle-France. Elle s'y développe lentement jusqu’à sa capture par les Britanniques en septembre 1760, qui marque la prise de contrôle de la ville par des élites politiques et économiques anglophones. Montréal n'en connaît pas moins une grande prospérité écono-

19. L'historienne Fernande Roy a consacré un article assez fouillé à ce répertoire plus hagiographique qu'historique et aux personnages - les héros et les héroïnes de Ville-Marie - qui en ont émergé d'une époque à l'autre. Voir Fernande Roy, «Une mise en scène de l'histoire. La fondation de Montréal à travers les siècles", Revue d'histoire de l'Amérique française, 46,1 (été 1992): 7-36.

20. Mentionnons la retranscription du discours prononcé par l'abbé Lionel Groulx à l'Académie Querbes en décembre 1939, où il reprend le récit de la fondation légendaire de VilleMarie: Lionel Groulx, Ville-Marie, joyau de l'histoire coloniale, 1642-1942 (Montréal, Commission du Troisième Centenaire de Montréal, 1940), 24 p. Ainsi qu'un texte du recteur de l'Université de Montréal intégré au compte rendu des fêtes et reproduisant lui aussi ce récit des origines: Olivier Maurault p.s.s., «Montréal en 1642», dans J.-P. Héroux, op. cit., 23-27. 
mique et, au milieu du $\mathrm{xIX}^{\mathrm{e}}$ siècle, les premières institutions municipales modernes prennent forme. La ville obtient ainsi une première charte municipale en $1833^{21}$. À la fin du siècle, grâce à son développement démographique et économique rapide, Montréal est devenue la métropole incontestée du Canada ${ }^{22}$. Ses élites économiques en grande partie anglophones dominent les activités financières et commerciales du pays ainsi que son vaste réseau de transport. Du point de vue identitaire et économique, la grande majorité des francophones de la ville existe en marge de cette Montréal-métropole dont le regard embrasse avant tout l'espace canadien ou nord-américain ${ }^{23}$. Dans ce cadre, le choix d'une commémoration de la fondation missionnaire, catholique et française de Ville-Marie par Maisonneuve, l'incarnation chevaleresque de ces thèmes, rend difficile - mais pas nécessairement impossible ${ }^{24}$ - l'intégration aux fêtes des éléments anglophones et non catholiques de la ville et, au contraire, sert la cause d'une réappropriation symbolique de l'espace urbain par la communauté franco-catholique.

Au-delà de ce dessein, dans le contexte d'une historiographie francophone mettant l'accent sur la période coloniale, la fondation de la ville par Maisonneuve s'impose presque naturellement dans le récit historique du développement de Montréal. L’obtention de la charte municipale de 1833 aurait pu servir de moment rassembleur. Sa révocation en 1836, en raison des troubles politiques que connaît le Bas-Canada et des soupçons qui pèsent sur les administrateurs de la ville, l'en empêche ${ }^{25}$. C'est d'ailleurs un fait qu'on s'empressera de souligner à Toronto. Contrairement à Montréal, la ville de Toronto a conservée sa charte, ce qui

21. Dickinson et Young parlent, pour les années 1840 et 1850, d'une "restructuration de la vie publique canadienne». John A. Dickinson et Brian Young, Brève histoire socio-économique $d u$ Québec (Sillery, Septentrion, 1995), 123.

22. P.-A. Linteau, op. cit., 36-38.

23. "Conçue pour embrasser de larges horizons, Montréal est excentrée dans un espace provincial qu'elle écrase de sa masse.» Gilles Sénécal et Claude Manzagol, "Montréal ou la métamorphose des territoires ", Cahiers de Géographie du Québec, 37,101 (septembre 1993): 351.

24. On peut se référer ici aux célébrations de 1908 du tricentenaire de la fondation de Québec par Samuel de Champlain. Certains des organisateurs, en particulier le gouverneur général du Canada Earl Grey, sont parvenus à enrichir ce moment premier d'un éventail d'autres figures, d'autres symboles et finalement d'autres valeurs que celles auxquelles on aurait pu s'attendre. Ainsi, la bataille des plaines d'Abraham, celle de Sainte-Foy, la bonne entente existant entre la France et l'Angleterre, le rôle du Canada dans l'empire britannique se bousculent sur la "scène commémorative» et éclipsent, au grand dam de certains francophones, la présence du fondateur de Québec. Voir H. V. Nelles, op. cit., 64-84.

25. Michèle Dagenais, La démocratie à Montréal. De 1830 à nos jours (Montréal, Ville de Montréal, 1992), 11. 
en fait, aux yeux des organisateurs, la première véritable municipalité d'Amérique du Nord britannique ${ }^{26}$. Ainsi, alors que les Montréalais se tournent vers des origines lointaines aux tonalités légendaires et insistent sur le rôle missionnaire unique de Ville-Marie en Amérique du Nord, les Torontois mettent l'accent sur la mise en place, plus récente, des institutions municipales qui ont donné naissance à la Toronto moderne.

Connue initialement sous le nom de York, la ville de Toronto est fondée en 1793 par le premier lieutenant-gouverneur du Haut-Canada, John Graves Simcoe ${ }^{27}$. Occupé sporadiquement par le passé, l'emplacement a été choisi par le nouveau lieutenant-gouverneur en raison de sa position stratégique avantageuse par rapport à l'ennemi américain. Conservateur, Simcoe rêve d'implanter en Amérique du Nord britannique une communauté qui sera contraire à la république américaine qu'il abhorre, c'est-à-dire une société dont le développement serait guidé par une classe dirigeante entièrement soumise à la Couronne britannique et à l'Église d'Angleterre ${ }^{28}$. L'influence de Simcoe se fera sentir au HautCanada et en particulier à York bien après son départ. Malgré sa position défensive avantageuse, la ville connaît l'épreuve du feu à deux occasions durant la guerre de 1812. Les habitants de York n'ont alors plus aucune sympathie pour la république américaine voisine ${ }^{29}$. L'arrivée massive d'immigrants britanniques dans les années 1820, dans le sillage de la chute de l'empire napoléonien, et le "raffinement» d'une idéologie impérialiste, en opposition à l'idéologie américaine, confirment le visage britannique de la colonie, et de la ville qui s'en veut le cour. L'approvisionnement des garnisons britanniques, puis les vagues successives d'immigrants européens, font en sorte que la ville s'impose comme centre politique et économique du Haut-Canada ${ }^{30}$. La ville connait alors, à l'instar de Montréal, une restructuration de sa vie publique et de ses institutions, restructuration dont la charte municipale de 1834 et le nom «Toronto» ne sont que deux manifestations.

Les Rébellions de 1837 n’ont trop affecté Toronto même si son premier maire, William Lyon Mackenzie, était le principal leader des insur-

26. CTA, RG 250, série 1, boîte 11, dossier 12, lettre de H. R. Alley à Arthur Sauvé datée du 9 novembre 1932.

27. J. M. S. Careless, Toronto to 1918: An Illustrated History (Toronto, J. Lorimer, 1983), 19.

28. Randall White, Ontario 1610-1985: A Political and Economic History (Toronto, Dundurn Press, 1985), 67.

29. J. M. S. Careless, op. cit., 33.

30. "York vigorous growth $[\ldots]$ no less strained its established framework and raised new public needs", ibid., 33, 43, 51. 
gés. Ces événements témoignent d'ailleurs de l'existence de courants réformistes qui sont, eux aussi, nourris par l'immigration britannique. Durant la deuxième moitié du siècle, la ville poursuit son développement comme centre commercial et administratif de la province sous la houlette d'élites surtout marchandes et, à partir des années 1870, s'industrialise rapidement. Ce développement rapide laisse deviner que Montréal et Toronto seront éventuellement nez à nez, puis qu'une seule s'imposera comme cœur du jeune État canadien. Tourné vers cet avenir en apparence pas si lointain, dans quel passé l'imaginaire collectif des Torontois peut-il se reconnaitre? Quelle place la fondation de York y occupe-t-elle?

Le centenaire de cet événement est timidement souligné à Toronto en 1893, mais c'est le cinquantenaire de l'incorporation de la municipalité de Toronto en 1884 qui est l'objet des festivités les plus élaborées ${ }^{31}$. Il faut toutefois souligner qu'il coïncide avec le centenaire de l'arrivée des Loyalistes au Haut-Canada. En conséquence, «[the] Loyalist centennial was a far wider feature of 1884 than Toronto's own anniversary ${ }^{32}$ ». Les Canadiens d'origine britannique représentent alors presque $95 \%$ de la population de la ville mais, malgré les efforts des Loyalistes, l'élitisme et les valeurs traditionalistes des organisateurs s'accommodent mal avec la cité moderne et industrielle que devient Toronto. Cette opposition contribue à un semi-échec des célébrations ${ }^{33}$. Le récit entourant l'exode loyaliste et la fondation de York ne représente donc plus, pour les élites commémorantes torontoises de 1934, un élément de la mémoire collective qui doit ou peut être cultivé. La fondation législative et institutionnelle de la cité fournira aux «faiseurs d'identité » un passé plus pertinent et plus apte à contribuer à la constitution d'un pôle identitaire consensuel ou fonctionnel.

La finalité de la mémoire collective est de fournir les éléments premiers de l'identité: le sens d'être singulier ou d'appartenir à une communauté unique aux traits relativement constants, distinctifs et, plus souvent qu'autrement, supérieurs ${ }^{34}$. Dans le contexte d'un renforcement

31. N. Knowles, op. cit., 67-90.

32. J. M. S. Careless, "The First Hurrah. Toronto's Semi-Centennial of 1884 », dans Victor L. Russell, dir., Forging a Consensus. Historical Essays on Toronto (Toronto, University of Toronto Press, 1984), 150.

33. Les manœuvres politiques et idéologiques entourant l'organisation et l'exécution de ces célébrations sont couvertes plus en détails dans N. Knowles, op. cit., 67-90.

34. Anthony P. Cohen, The Symbolic Construction of Community (Londres, Routledge, 1985), 12. 
ou d'une redéfinition de l'identité collective, la fondation joue le rôle de moment premier, c'est-à-dire de point de départ ou de référence pour la communauté où se reconnaissent ses membres. Confrontées à des passés et à des contextes particuliers, les élites commémorantes, à Montréal comme à Toronto, se tournent vers des moments premiers qui répondent à leurs sensibilités et à leurs aspirations.

Concrètement, ce processus de sélection et d'interprétation du passé ne représente qu'un aspect mineur de la mécanique administrative mise en place pour l'organisation et pour l'exécution des fêtes étudiées. À travers un survol de la constitution et de l'évolution des groupes chargés de l'organisation des deux séries de célébrations, des programmes qu'ils ont proposés et des résultats auxquels ils ont abouti, j'espère mettre en lumière cette mécanique, sa dynamique particulière et le fossé - étroit dans un cas, considérable dans l'autre - séparant les intentions de l'exécution.

\section{LE PERSONNEL ET LES OBJECTIFS}

Selon Alan Gordon, durant la période qui s'étend de la fin du $\mathrm{xIX}^{\mathrm{e}}$ siècle à la crise économique des années 1930, les élites commémorantes (heritage elites) montréalaises, au départ surtout composées de philanthropes et d'individus rattachés au milieu local, sont de plus en plus encadrées par des institutions fédérales ou provinciales. Cette transition se fait dans le cadre d'une redéfinition progressive du rôle de ces dernières qui prennent progressivement en charge la mémoire collective ou publique de la nation ${ }^{35}$. On verra bientôt que cette transition transparaît dans le cas des célébrations étudiées. Je vais donc identifier dans les pages qui suivent les principaux individus qui sont à l'origine de l'organisation des fêtes du centenaire de l'incorporation de Toronto et du tricentenaire de Montréal, voir qui ils sont et quels groupes ils représentent.

L’idée de célébrer les anniversaires étudiés est attribuée aux maires du moment: William Stewart pour Toronto, Adhémar Raynault pour la Montréal. Stewart aurait déjà proposé le projet alors qu’il était échevin

35. A. Gordon, op. cit., xv. Notons que ce processus de redéfinition est déjà bien en train au niveau des autorités municipales qui ont en quelque sorte ouvert la voie. Voir les articles de Michèle Dagenais pour des exemples concrets de cette redéfinition dans la sphère culturelle: Michèle Dagenais, "Vie culturelle et pouvoirs publics locaux. La fondation de la bibliothèque municipale de Montréal", Urban Historical Review/Revue d'histoire urbaine, 24,2 (mars 1996): 4056; Michèle Dagenais, «Entre tradition et modernité. Espaces et temps de loisir à Montréal et Toronto au xx ${ }^{e}$ siècle", Canadian Historical Review, 82,2 (juin 2002): 308-330. 
à la fin des années $1920^{36}$, le ressuscitant une fois devenu maire en 1931 . Stewart n'a pas bonne presse dans l'historiographie torontoise. Conservateur lié au parti fédéral de Bennett, son seul mandat à la tête de la ville ne laisse pas une forte impression et son seul mérite semble d'avoir maintenu la ville à flot durant les premières années de la crise ${ }^{37}$. Du côté de Montréal, l'idée de célébrer le tricentenaire de Montréal apparaît dans le programme électoral d'Adhémar Raynault lors de la campagne électorale de 1936. Il y voit un moyen d'éveiller la fierté des citoyens pour leur ville. Simultanément maire et député provincial, sous la bannière de l'Union Nationale, Raynault fait amender en 1937 la Charte municipale de Montréal pour pourvoir à la création de la "Commission du III ${ }^{e}$ Centenaire ${ }^{38}$ ». Compromis dans un scandale financier, Raynault doit entre-temps quitter la mairie en 1938, mais les fameuses déclarations du maire réélu Camillien Houde sur la conscription lui permettent de revenir au pouvoir en 1940. Puisque Montréal est à ce moment sous tutelle, Raynault fait figure de "maire honorifique» et les fêtes du tricentenaire, dont il est le président d'honneur, deviennent une de ses principales «responsabilités ${ }^{39}$ ». Cela dit, les deux maires ont peu de pouvoir dans l'organisation des festivités. Les comités qui sont mis en place sont chargés de les préparer, et surtout c'est à leurs directeurs que revient la majeure partie du travail.

Dès le $1^{\text {er }}$ juin 1931, Stewart propose qu’un comité formé des échevins de la municipalité choisisse les membres du comité des fêtes. Composé de représentants de "various organizations of the citizens of Toronto", il sera responsable du programme des fêtes et de son exécution ${ }^{40}$. Ce Toronto Centennial Celebrations Committee (TCCC) sera dirigé à partir du 13 octobre 1931 par le lieutenant-colonel Herbert R. Alley ${ }^{41}$. À Montréal, une loi sanctionne la création de la Commission du Troisième

36. CTA, RG250, série 1, boîte 1, dossier 1, manuscrit inédit intitulé A Century Passes. 1834 Toronto - 1934. A Record of Many Epochal Events During City's Centennial Year Celebration [apparemment de la main de Herbert R. Alley], 2.

37. J. Lemon, op. cit., 74.

38. Victor Morin, "Préface», dans J.-P. Héroux, op. cit., 10.

39. «Il ne peut même pas remplacer la femme de ménage de son bureau sans l'approbation de la Commission municipale.», Claude-V. Marsolais, «Adhémar Raynault, l'homme à l'œillet, répare les pots cassés ", dans Claude-V. Marsolais, Luc Desrochers et Robert Comeau, Histoire des maires de Montréal (Montréal, VLB éditeur, 1993), 254.

40. CTA, Toronto City Council Minutes, 1931, appendice A, 1819.

41. CTA, RG5, boîte 189, rapport du greffier de la ville, Roy V. Henderson au Steering Committee on Sesquicentennial Celebrations intitulé Toronto Centennial Celebrations - 1934 (With Notes on Semi-Centennial - 1884 and $125^{\text {th }}$ Anniversary - 1959) daté du 2 novembre 1979, 3. 
Centenaire de Montréal (CTCM) le 20 mai 1937. Cette dernière est composée de «certains membres du conseil [municipal], de représentants de divers corps publics et, au besoin, d'autres personnes pour élaborer un programme de fêtes, manifestations ou démonstrations ${ }^{42}$ ». Le maire nomme Léon Trépanier au poste de directeur général des fêtes ${ }^{43}$.

Les archives du TCCC sont dominées par l'importante correspondance du directeur des fêtes qui témoigne de sa gestion méticuleuse des différents aspects des célébrations. Descendant des premiers loyalistes et militaire de carrière, Alley est toujours à la tête du Toronto Regiment au moment de sa nomination ${ }^{44}$. La littérature militaire nous donne quelques indications quant à la relation qu'il entretient avec Stewart: peu avant sa nomination au poste de directeur des fêtes, Alley a fait appel au maire au nom des officiers de son régiment pour qu'il intervienne auprès de l'administration Bennett à Ottawa, qui s'apprête à sabrer dans les budgets d'entraînement ${ }^{45}$. Stewart parvient à ouvrir les portes du bureau du Premier ministre à Alley et à ses collègues. Ils obtiendront d'importantes concessions financières. Autrement, les sources ne révèlent rien dans l'expérience passée de Alley qui en fasse un candidat particulièrement habilité à prendre en charge les célébrations du centenaire.

Ce n'est pas le cas de Léon Trépanier. diplômé de l’Université d'Ottawa en études classiques, il est journaliste à La Presse. À partir de 1920, il s'engage en politique municipale en tant que membre d'une commission provinciale chargée d'étudier un nouveau mode d'administration pour Montréal, puis comme échevin tout au long des années 1920 et 1930. Il sera également président de la Société Saint-Jean-Baptiste (SSJB) de 1925 à 1929 et contribuera à l'érection de la croix du mont Royal ainsi qu'à la mise en train d'imposants défilés pour les fêtes de la Saint-Jean-Baptiste. Dans les années 1920, il a également visité un grand nombre de villes nord-américaines et européennes pour y observer les expositions et les fêtes commémoratives. Finalement, en 1935, il organise les fêtes du tricentenaire de Trois-Rivières, en 1937 celles du

42. V. Morin, loc. cit., 10.

43. C.-V. Marsolais, loc. cit., 253; V. Morin, loc. cit., 10.

44. CTA, RG250, série 1, boîte 2, dossier 3, lettre du 10 juin 1932 du Board of Control (City Clerk) au maire; et CTA, RG250, série 1, boîte 1, dossier 1, manuscrit inédit intitulé A Century Passes. 1834 - Toronto - 1934. A Record of Many Epochal Events During City's Centennial Year Celebration [apparemment de la main de Herbert R. Alley], 2.

45. Donald James Goodspeed, Battle Royal: A History of the Royal Regiment of Canada 18621962 (Toronto, Royal Regiment of Canada, 1962), 335. 
centenaire de Sherbrooke et l'année suivante celles du centenaire du Saguenay ${ }^{46}$. Doyen du conseil municipal, il démissionne en 1938 de son poste d'échevin pour prendre la tête de la $\mathrm{CTCM}^{47}$.

Sans procéder à une prosopographie extensive des membres de la CTCM et du TCCC, il est possible d'étudier sommairement la composition de ces comités. Le comité général torontois compte dix-huit membres. En plus du directeur, on y retrouve des représentants des principaux quotidiens de la ville ${ }^{48}$, de différents organes gouvernementaux municipaux et provinciaux, des associations de chefs d'entreprises, des syndicats, des principales expositions abritées par la ville, ainsi que d'un délégué de l'University of Toronto. Il est composé exclusivement d'hommes d'origine anglo-saxonne mais, tel qu'il a été demandé, il est largement représentatif des principaux corps sociaux torontois.

Le comité général montréalais, présidé par le maire de la ville, compte quant à lui douze membres. On y remarque la présence prédominante de membres de l'élite économique et politique canadienne-française de Montréal. Parmi ceux-ci, Victor Morin, qu’Alan Gordon considère comme "the figure to most influence public memory in Montreal ${ }^{49}$ ». Le comité inclut également trois anglophones: l’orfèvre Henry-G. Birks, le conseiller municipal Frederick-B. Todd ainsi que T. Taggart Smyth, président de la Ligue du Progrès Civique et gérant-général (directeur) de la Banque d’Épargne de la Cité et du district de Montréal, qui occupe la fonction de trésorier auprès de la CTCM. En termes ethno-linguistiques, la commission est représentative des différentes composantes culturelles de la population montréalaise ${ }^{50}$, mais compte tenu de l'influence économique et culturelle encore appréciable exercée par la minorité anglophone sur Montréal, on observe un certain désengagement des anglophones. Désengagement qui s'explique certainement par le rôle

46. Ces informations sont tirées de Les biographies françaises d'Amérique (Montréal, Journalistes associés, 1942), 629; et de R. Tourangeau, op. cit., 72.

47. Notons que si Morin insiste sur les compétences de Trépanier (V. Morin, loc. cit., 11), qui sont évidentes, Marsolais, dans son texte sur le maire Raynault, affirme que la nomination de Trépanier est avant tout un geste politique destiné à écarter un rival potentiel du conseil municipal. C.-V. Marsolais, loc. cit., 253.

48. Il s'agit du Toronto Daily Star, du Globe, du Evening Telegram et du Mail and Empire. CTA, RG5, boîte 189, rapport du greffier de la ville, Roy V. Henderson, au Steering Committee on Sesquicentennial Celebrations intitulé Toronto Centennial Celebrations - 1934 (With Notes on SemiCentennial - 1881 and 125th Anniversary - 1959) daté du 2 novembre 1979, 2.

49. A. Gordon, op. cit., 56.

50. En 1941, les francophones représentent $66,3 \%$ de la population de la ville de Montréal, contre $20,3 \%$ pour les anglophones d'origine britannique. P.-A. Linteau, op. cit., 318. 
prédominant de l'Église catholique et par l'accent mis sur la fondation française et catholique de Montréal. On peut aussi lier en partie ce mouvement à un effacement plus large de ce groupe de la mémoire collective partagée et commémorée par les Montréalais à partir des années $1920^{51}$. Notons enfin que le comité montréalais est aussi composé exclusivement d'hommes ${ }^{52}$.

Que tirer d'une comparaison entre ces deux groupes? Premièrement, il faut souligner les débuts similaires des préparatifs des célébrations et de la mise sur pied des commissions. De même, l'influence des deux directeurs se fera rapidement sentir sur l'orientation des festivités. Toutefois, si le TCCC se rend au terme de son mandat, la CTCM connaîtra des ratés considérables à la suite de la Crise, mais surtout de la guerre qui la prive de tout moyen de mettre en œuvre, même partiellement, son programme. Dans ce contexte, les membres du comité chargé de l'aspect religieux des fêtes montréalaises prendront l'initiative, transformant substantiellement le contenu des fêtes.

\section{LES PROGRAMMES}

Les deux comités préparent différentes esquisses de programme. Les modifications successives d'une esquisse à l'autre illustrent les itinéraires parfois sinueux de ces projets de commémoration. La distance est quelquefois assez prononcée entre les ambitions de départ des organisateurs et les programmes finaux qui déterminent le déroulement des festivités.

À Toronto, un premier document est produit en 1931 et soumis au conseil municipal par le comité spécial sur les célébrations du centenaire, ancêtre du TCCC. On y recommande la tenue d'un grand nombre d'activités réparties durant toute l'année selon une série de thématiques. Après des célébrations d'ouverture le 6 mars, date anniversaire du centenaire de l'incorporation, se succéderaient des célébrations religieuses, patriotiques, sportives, "fraternelles", ouvrières, agricoles et militaires. Ces activités correspondent à des fêtes préexistantes. Ainsi, les fêtes religieuses coïncideraient avec Pâques, les fêtes patriotiques avec le Victoria Day, les fêtes sportives avec le Dominion Day, les festivités ouvrières

51. A. Gordon, op. cit., 178-183.

52. Dans une lettre adressée au directeur des fêtes, Thérèse F. Casgrain se plaint d'ailleurs de cet état de fait, soulignant que : "Si l'on veut les [les Montréalaises] glorifier, il serait logique, me semble-t-il, de commencer par leur accorder la place qui leur revient dans notre société moderne. " DGDA, VM12, série 2, dossier 17, lettre de Thérèse F. Casgrain à Léon Trépanier datée du 12 janvier 1939. 
avec la fête du Travail, les fêtes agricoles avec le Thanksgiving et les festivités militaires avec l'Armistice ${ }^{53}$. Les organisateurs espèrent ainsi réduire les dépenses, tout en profitant de l'attrait de fêtes annuelles déjà très populaires.

De plus, l'attachement à l'empire est clairement souligné dans le texte du comité spécial. L’espoir de pouvoir bénéficier de la présence d'invités qui lui sont associés ("outstanding men of the Empire») et capables d'illuminer de leur renom les festivités se manifeste dans le dernier paragraphe. Les membres du comité spécial espèrent particulièrement une visite du prince de Galles ${ }^{54}$.

Puisque les nuages de la crise économique tardent à se dissiper au cours des deux années suivantes, le TCCC se résigne à restreindre son programme. Il substitue à des fêtes étendues sur toute l'année des cérémonies d'ouverture en mars - qui auront surtout une fonction publicitaire ${ }^{55}$ - et trois «moments forts». Ces trois périodes de trois jours seront tout de même arrimées à des fêtes: la première coïncide avec le Victoria Day, la seconde avec le Dominion Day et la dernière avec le "civic holiday» de Toronto. Notons l'association de chacune de ces dates à un cadre identitaire particulier: empire, nation et cité. La première série de fêtes tourne d'ailleurs autour de la thématique impériale et compte largement sur la visite de dignitaires britanniques. L'ambiguiité des sources quant à l'identité de ce ou de ces visiteurs tient aux difficultés qu'aura le TCCC à attirer de tels dignitaires à Toronto ${ }^{56}$. Les fêtes de juillet sont sensées constituer l'apogée du centenaire et devront, selon les recommandations du comité général des fêtes, avoir un

53. CTA, Toronto City Council Minutes, 1931, appendice A, 1820.

54. Ce groupe inclut en particulier les individus ayant été, dans le passé, gouverneurs généraux du Dominion of Canada. CTA, Toronto City Council Minutes, 1931, appendice A, 1820.

55. CTA, RG250, série 1, boîte 2, dossier 3, lettre du 20 mars 1933 du comité général des fêtes au maire et au conseil municipal concernant le programme soumis aux deux instances par $\mathrm{H}$. R. Alley.

56. Cet intérêt du comité général des fêtes est évident: «We particularly commend the idea of bringing outstanding personnages to Toronto, and consider that this will be an important factor in attracting visitors to the City. »CTA, RG250, série 1, boîte 2, dossier 3, lettre du 20 mars 1933 du comité général des fêtes au maire et au conseil municipal concernant le programme soumis aux deux instances par H. R. Alley. Et le malaise de Alley, qui n'arrive pas à attirer de personnage important, à peine voilé dans cette entrée de sa proposition de programme: «Reception of Distinguished Personnages who cannot now be named. » CTA, RG250, série 1, boîte 2, dossier 3, lettre de H. R. Alley du 20 mars 1934 au maire et au conseil municipal contenant sa proposition de programme et le budget qui lui est lié. 
"provincial tone ${ }^{57}$ ». Les fêtes d'août sont centrées sur la grande réunion des vétérans du Canadian Corps. Un programme officiel basé sur ce canevas est présenté au début de 1933 et adopté en septembre par le conseil municipal ${ }^{58}$.

Malgré le contexte économique difficile ${ }^{59}$, ce programme sera appliqué dans sa totalité. Avec un budget qui atteindra éventuellement $173000 \$$, fournis presque entièrement par la ville, auquel il faut ajouter l'aide ponctuelle du secteur privé ${ }^{60}$, les fêtes du centenaire de Toronto connaissent un déroulement relativement serein. Avec un budget final qui ne dépassera pas $35000 \$$ et un programme sévèrement amputé, Montréal offre en contraste l'image de cérémonies qui ont, dans une certaine mesure, déraillé ${ }^{61}$. Les intentions initiales exprimées par ses organisateurs suggèrent toutefois que, dans un contexte différent, les célébrations du tricentenaire de Montréal auraient ressemblé à celles de Toronto.

On peut en grande partie reconstituer ces intentions initiales à partir des documents de travail élaborés par Léon Trépanier avant sa démission. Il est évidemment impossible de déterminer si les festivités auraient vraiment eu ce visage, mais on peut supposer que les grandes lignes du programme auraient été respectées.

Les célébrations prévues pour 1942 devaient remplir deux objectifs: être, pour les visiteurs comme pour les habitants de la ville, une précieuse leçon d’histoire, mais aussi, permettre de «réintégrer Montréal [...] comme le grand centre touristique au Canada ${ }^{62} »$. Pour concilier ces

57. CTA, RG250, série 1, boîte 2, dossier 3, lettre du 20 mars 1933 du comité général des fêtes au maire et au conseil municipal concernant le programme soumis aux deux instances par $\mathrm{H}$. R. Alley.

58. CTA, RG5, boîte 189, rapport du greffier de la ville, Roy V. Henderson au Steering Committee on Sesquicentennial Celebrations intitulé Toronto Centennial Celebrations - 1934 (With Notes on Semi-Centennial - 1884 and 125th Anniversary - 1959) daté du 2 novembre 1979, 9.

59. "Every means of economizing was sought, found and applied, consistent with success.", CTA, RG250, série 1, boîte 1, dossier 1, manuscrit inédit intitulé A Century Passes. 1834 - Toronto - 1934. A Record of Many Epochal Events During City's Centennial Year Celebration [apparemment de la main de Herbert R. Alley], 2. "You will observe that many things have been omitted from the programme which if general conditions were better would undoubtly be included.», CTA, Toronto City Council Minutes, 1934, appendice C, 29.

60. Par exemple, la compagnie Eaton collaborera au financement de certaines installations liées à la réunion des vétérans du Canadian Corps. CTA, RG 250, série 1, boîte 5, dossier 5, copie des reçus pour des dons d'entreprises privées au TCCC.

61. C.-V. Marsolais, loc. cit., 254.

62. DGDA, VM12, série 0, dossier 3, Autorisation de la Législature provinciale, 1937, 2. 
deux objectifs de nature très différente, Trépanier parle d’une «exploitation intelligente de notre passé historique ${ }^{63}$ ». On voit comment, à la base, les considérations idéologiques ou mémorielles doivent coexister avec des impératifs d'ordre économique.

D’ailleurs, dans le cadre du programme proposé par Trépanier, il ne peut pas y avoir de malentendu quant au public touristique visé. Le directeur des fêtes a les yeux tournés vers le Sud:

je dis qu'en raison des événements empreints de splendeur et de dignité qui marqueront le Trois-centième [sic] Anniversaire de la Fondation de Montréal en 1942, des efforts tout particuliers devraient s'exercer dans le but de faire connaitre davantage le Montréal-historique aux cent vingt millions d'habitants de la République américaine ${ }^{64} \ldots$

Trépanier met l'accent sur les «liens historiques» qui uniraient plus de la moitié des États américains à Montréal et sur l’importance de les mettre en lumière. Les fêtes seront donc ouvertes sur le monde en vue d'attirer à Montréal des touristes d'Europe et du reste du Canada, mais aussi de rassembler sur la place publique hommes et femmes de toutes croyances et permettre aux «deux grandes nationalités [de] se glorifier» mutuellement ${ }^{65}$. Trépanier espère également ramener à Montréal les "Anciens", c'est-à-dire les Montréalais dispersés un peu partout sur le globe et «se glorifiant» d'y être nés.

De quoi auront donc l'air ces fêtes? Se référant à l'exemple newyorkais $^{66}$, le directeur souligne qu'elles passeront d'abord par un important effort d'embellissement de Montréal. Selon Trépanier, l'apparence "actuelle» (1937) est indigne du prestige dont il souhaite draper la ville à l'occasion du tricentenaire. Autre lacune dénoncée par le directeur: les infrastructures culturelles. Puisque que les festivités laisseront beaucoup de place aux artistes locaux, Trépanier espère laisser un auditorium municipal comme contribution permanente des fêtes à la ville. Le

63. Dans les lignes qui suivent, il pousse plus loin et souligne que : «On a souvent proclamé en chansons et en écrits que notre histoire est un trésor inestimable. Si cela est vrai, ce ne serait pas en diminuer la valeur que de l'interpréter de façon à ce qu'elle nous apporte des revenus en argent. » DGDA, VM12, série 0, dossier 3, Autorisation de la Législature provinciale, 1937, 3.

64. DGDA, VM12, série 0, dossier 3, Autorisation de la Législature provinciale, 1937, 3.

65. DGDA, VM12, série 0, dossier 3, Autorisation de la Législature provinciale, 1937, 7.

66. En 1938, New York accueille l'Exposition mondiale et, pour l'occasion, s'est lancée dès 1937 dans une vaste campagne de nettoyage et d'embellissement de la ville, utilisant des milliers de chômeurs de la ville. DGDA, VM12, série 0, dossier 3, Autorisation de la Législature provinciale, 1937,5 . 
programme comportera aussi certaines composantes religieuses ${ }^{67}$. De plus, le directeur des fêtes veut faire de l'anniversaire de la ville un tribut «à notre jeunesse, $[\mathrm{au}]$ triomphe de la jeune génération sur l'oisiveté forcée qui l'opprime ${ }^{68} »$. Les sports, les aspects militaires de l'histoire montréalaise et l'artisanat local sont également des thématiques qui seront privilégiées. L'artisanat intéresse particulièrement le directeur puisqu'il donne aux célébrations un accent folk apprécié des touristes, tout en assurant des revenus aux artisans et aux fêtes ${ }^{69}$. Alan Gordon s'est penché sur ce phénomène:

[t]hreats to the character of place often, paradoxically, involved an entrenchment of «distinctive» cultural features. [...] Hand-sewn quilts and needlework represented a revival of old ways and a rejection of industrial modernity in an effort to satisfy an urban demand for authentic, rustic artifacts ${ }^{70}$.

Cet intérêt pour l'artisanat relèverait donc autant d'un opportunisme économique ("an attempt to construct tourist experiences ${ }^{71}$ ) que d'un malaise plus général face au déraillement de l'économie industrielle et capitaliste.

Ce programme présente des ressemblances frappantes avec celui mis en œuvre à Toronto: thématiques, objectifs et activités sont les mêmes Les célébrations des deux villes ont suivi, du moins durant les premières étapes de leur préparation, des itinéraires similaires où ne paraissent pas de différences fondamentales entre les sensibilités des élites "commémorantes» canadiennes-françaises et canadiennes-anglaises. Compte tenu des relations que les deux directeurs ont entretenues avec les organisateurs de célébrations du genre en Europe et en Amérique du Nord ${ }^{72}$, on peut suggérer qu'il y a là diffusion d'un certain modèle commun, axé sur la promotion du tourisme. Les différences majeures résultent surtout d'un contexte particulier.

67. DGDA, VM12, série 0, dossier 3, Autorisation de la Législature provinciale, 1937, 7.

68. DGDA, VM12, série 0, dossier 3, Autorisation de la Législature provinciale, 1937, 8.

69. DGDA, VM12, série 0, dossier 3, Autorisation de la Législature provinciale, 1937, 9.

70. A. Gordon, op. cit., 13. On pourra se référer aussi, plus largement, aux travaux de Ian McKay, The Quest of the Folk: Antimodernism and Cultural Selection in Twentieth-Century Nova Scotia (Montréal, McGill-Queens University Press, 1994), 371 p.

71. A. Gordon, op. cit., 13.

72. Comme en témoigne pour Trépanier Les Biographies françaises d'Amérique. Dans le cas d'Alley, on se rapportera à CTA, RG 250, série 1, boîtes 5 et 6 se référant aux relations qu'entretient Alley avec des organismes «commémorants» de Glasgow et de différentes villes des États-Unis à partir du moment de sa nomination. 
Le sort des fêtes du tricentenaire est remis en question par la Deuxième Guerre mondiale. Malgré le climat incertain, Léon Trépanier continue d'appuyer la tenue de fêtes pour l'anniversaire de Montréal jusqu'au mois d'octobre 1940, mais à condition que les gouvernements provincial et fédéral lui apportent leur support ${ }^{73}$. Ce ne sera pas le cas. Le 26 novembre 1940, soulignant que «[p]lusieurs corporations importantes, ainsi que les autorités fédérales, provinciales et municipales semblent d'opinion qu'il vaut mieux ne pas continuer la préparation de ces fêtes $^{74}$ », Trépanier démissionne. Quel poids l'internement du maire Camillien Houde et l'ouverture du poste de maire, auront-ils dans cette décision? Difficile de le déterminer mais, comme l'avait craint Adhémar Raynault, lui et Trépanier croisent le fer brièvement dans l'arène municipale. Défait par une mince majorité des voix, l'ancien directeur des fêtes deviendra attaché spécial à l'adjudant-général de l’armée et directeur civil conjoint du recrutement pour le Canada.

En 1942, dans une lettre circulaire envoyée à la majorité des organismes et institutions francophones et anglophones de la ville associés aux célébrations du tricentenaire, on annonce qu'en raison des conditions de guerre, aucune des activités prévues n'aura lieu. La commission les invite toutefois à tenir leurs propres activités afin de souligner humblement l'occasion au cours de l'année $\mathrm{e}^{75}$. Plusieurs organismes répondront à l'appel, mais une seule institution s'imposera clairement: l'Église catholique. Par l'entremise du comité des fêtes religieuses, dirigé par $\mathrm{M}^{\mathrm{gr}}$ Joseph Charbonneau et auquel sont attachés une douzaine de clercs dont Joseph-Papin Archambault s.j., Olivier Maurault p.s.s. et Lionel Groulx, cette institution mènera les fêtes à bon port.

Ce comité semble, dès le départ, avoir occupé une place particulière au sein de la CTCM. Semi-autonome, il ne semble pas avoir eu de comptes à rendre au comité général, qui semble encore tout ignorer de ses intentions ou du programme de l'Église aussi tard qu'en $1941^{76}$. Ne

73. Comme en témoignent des articles du Montreal Daily Star («Tercentenary Decision Due next month") et de La Patrie ("Les fêtes de 1942 sont à la merci de Québec et d'Ottawa») datés du 18 octobre 1940 et conservés dans les archives de la CTCM. DGDA, VM12, série 2, dossier 31.

74. DGDA, VM12, série 2, dossier 17, lettre de Léon Trépanier au président et aux membres de la CTCM datée du 26 novembre 1940.

75. DGDA, VM12, série 2, dossier 34, lettre de Jean-Paul Héroux à la Saint-George Society of Montreal, datée du 2 février 1942.

76. DGDA, VM12, série 2, dossier 1, lettre d'Adhémar Raynault à Mgr Joseph Charbonneau, datée du 28 février 1941. 
dépendant pas du budget de la $\mathrm{CTCM}^{77}$, le comité des fêtes religieuses dirigera le gros des festivités liées au tricentenaire et y apposera sa marque. Le discours de cette importante composante de l'élite canadienne-française imposera plutôt aux festivités son cadre idéologique et culturel. À ses côtés, on retrouvera la présence significative des membres de la Chambre de commerce des jeunes de Montréal (CCJM) et de l'Association catholique de la jeunesse canadiennefrançaise (ACJC) ainsi que des "rescapés» de la CTCM. Bref, les célébrations seront dominées par des membres de la société canadiennefrançaise associés ou sympathiques aux courants traditionalistes du nationalisme canadien-français.

À l'origine, les deux séries de fêtes étudiées semblent donc partir de positions et d'intentions convergentes. À Montréal comme à Toronto, les programmes de base des fêtes sont centrés sur des thématiques similaires : célébration du passé de la ville à des fins pédagogiques mais aussi commerciales et touristiques. On y propose des festivités sportives, militaires, religieuses, culturelles et patriotiques s'adressant à la majeure partie de la communauté civique. Dans les deux cas, on espère favoriser la venue de touristes, surtout américains, par une campagne massive d'embellissement de la ville, mettant à l'ouvrage les milliers de chômeurs qu'a créés la crise économique. Le contexte persistant de la crise oblige les élites "commémorantes torontoises à restreindre leur programme à l'essentiel. Mais ces restrictions paraissent insignifiantes par rapport aux bouleversements provoqués à Montréal par la Deuxième Guerre mondiale. Après quelques mois d'hésitation, et devant le refus des gouvernements provincial et fédéral de l'appuyer, la CTCM doit se résoudre à une dissolution presque complète de son programme, laissant la voie ouverte à des fêtes surtout religieuses.

\section{LES FÊTES}

C'est ici que convergent (ou ne convergent pas dans le cas de Montréal) les contextes historiques des fêtes et les efforts préparatoires des différentes instances engagées. La présentation des deux séries de célé-

77. Même si, au cours des fêtes, le comité des fêtes religieuse à recours à la CTCM pour l'assister à balancer son budget final par quelques contributions légères (pour un total de $5500 \$$ ). «Nos finances seraient à flot et de nos cœurs monterait à flots pressés notre reconnaissance.» DGDA, VM12, série 6, sous-série 1, dossier 1, lettre de $\mathrm{M}^{\mathrm{gr}}$ Chaumont au maire Adhémar Raynault, datée du 16 juin 1942; voir aussi DGDA, VM12, série 6, sous-série 1, dossier 1, lettre de monseigneur Charbonneau à Jean-Paul Héroux, datée du 17 décembre 1942. 
brations sous la forme de brefs récits chronologiques, mettant l'accent sur les moments forts du programme, permettra de saisir le contraste important entre le cas montréalais et celui de Toronto.

Les cérémonies officielles du centenaire de l'incorporation de Toronto s'ouvrent le 5 mars 1934 par une soirée au Coliseum de Toronto. On y accueille plus de 12000 personnes dans le cadre d'un "civic nondenominational service ${ }^{78}$ ». Sermons, chants et prières se succèdent devant une assemblée où figurent une foule de dignitaires, dont R. B. Bennett et G. S. Henry, les premiers ministres fédéral et provincial, ainsi que le chef de l'opposition officielle à Ottawa (et petit-fils du premier maire de la ville) William Lyon Mackenzie King ${ }^{79}$. Sur le coup de minuit, un grand bûcher est allumé à l'extérieur et cent fusées «d'artifice» sont lancées dans le ciel torontois. Le lendemain, un grand dîner est organisé au King Edward Hotel. Les mêmes dignitaires y participent et plusieurs discours sont prononcés à l'occasion de toasts à la Ville-Reine. Comme dans le cas de la réunion du conseil de ville, ce dîner reste fermé à la majorité de la population torontoise, qui peut toutefois, en après-midi, assister à une cérémonie devant l'hôtel de ville où des gerbes de fleurs sont déposées devant le cénotaphe dédié aux morts de la Grande Guerre. De plus, une plaque où figurent les noms des maires et échevins de 1834 et 1934 est dévoilée près de l'entrée de l'hôtel de ville ${ }^{80}$.

Le 23 mai, le premier temps fort des fêtes s'ouvre en marge de la fête de la Reine (Victoria Day). Deux grands défilés sont organisés où on retrouve de nombreuses unités militaires (pour un total de 7500 soldats) et différents chars allégoriques sur lesquels sont rejoués les grands moments de l'histoire de la ville et du Dominion. Ce défilé est suivi de la cérémonie d'ouverture d'un Fort York restauré, qui fera figure de monument permanent des fêtes du centenaire ${ }^{81}$. Les célébrations de mai prennent fin, les 26 et 27 , avec une grande fête à laquelle participent les minorités ethniques de la Ville-Reine.

Juillet, le second moment fort des fêtes, en est aussi le cœur. On s'attend à ce que les activités permettent de réduire le déficit jusqu’à maintenant relativement élevé ${ }^{82}$. Les 2,3 et 4 juillet, un impressionnant

78. CTA, RG250, série 1, boîte 2, dossier 3, lettre de Alley à Stewart, le 20 mars 1933.

79. The Daily Star, le 6 mars 1934, 1.

80. The Daily Star, le 7 mars 1934, 1.

81. CTA, RG250, série 1, boîte 9, dossier 3, lettre de George H. Locke à C. H. Mitchell, le 20 janvier 1933.

82. CTA, RG250, série 1, boîte 2, dossier 3, lettre de J. A. Northey au maire et au Board of Control, le 20 mars 1933. 
"pageant» en douze tableaux intitulé "Milestones of a Century» est présenté sur le site de l'exposition nationale ${ }^{83}$. Le 2 , un grand déjeuner est aussi organisé au cours duquel le maire de Toronto accueille ses collègues d'une soixantaine de municipalités avoisinantes. Plus tard dans la journée, on célèbre à grands coups de défilés, d'activités sportives et de spectacles aériens le Dominion Day. Le 4 juillet, jour de la fête nationale américaine, sous les yeux de dignitaires et de contingents militaires venant des États-Unis, du Canada et du Royaume-Uni, un monument aux morts de la guerre de 1812 est élevé dans l'enceinte du Fort York ${ }^{84}$.

Les cérémonies officielles du centenaire se terminent avec la réunion des vétérans du Canadian Corps en août ${ }^{85}$. Dépassant de loin les objectifs fixés par les organisateurs, ces festivités débutent le 4 août par une cérémonie devant le cénotaphe situé près de l'hôtel de ville et se poursuivent par un grand défilé, auquel participent plus de 60000 vétérans canadiens, français, britanniques, amérindiens et allemands. Deux personnalités militaires britanniques, dont les liens avec le Canada sont assez ténus (pour ne pas dire inexistants), sont invitées d'honneur: le vicomte Allenby et sir Reginald Tyrwhitt ${ }^{86}$. Le lendemain, le dimanche 5 août 1934, est dédié à un service religieux en plein air. Les vétérans y font de nouveau serment de servir Dieu, le roi et leur pays ${ }^{87}$. Le dernier jour de la réunion est marqué par un grand rassemblement militaire dans l'amphithéâtre naturel de Riverdale Park. Plus de 50000 anciens combattants sont présents ainsi qu'environ 250000 civils. Le TCCC a rempli ses fonctions. Il est officiellement dissout le 10 août, mettant fin au programme officiel des fêtes.

Le 15 octobre 1941, en l'honneur de la première visite des fondateurs sur l'île de Montréal, les fêtes du tricentenaire de la fondation de la ville

83. Ironiquement, un des tableaux est consacré au défilé commémoratif de 1884, centenaire de l'arrivée des United Empire Loyalists (UEL) - commémoration de commémorants? CTA, RG250, série 1, boîte 1, dossier 1, A Century Passes. 1834 - Toronto - 1934. A Record of Many Epochal Events During City's Centennial Year Celebration, chapitre intitulé "Milestones of a Century» [manuscrit apparemment de la main d'Alley].

84. The Globe, le 5 juillet 1942, 4.

85. Cette partie des cérémonies a aussi été étudiée par Jonathan F. Vance, «"Today They Were Alive Again": The Canadian Corps Reunion of 1934 ", Ontario History, 87,4 (décembre 1995): 327-344.

86. The Globe, le 4 août 1934, 1. Alley avait tenté d'obtenir la présence de lord Byng, qui avait dirigé le Canadian Corps en Europe, mais pour des raisons de santé, celui-ci dut décliner. J. F. Vance, loc. cit., 332.

87. Le "Drumhead Service» accueille plus d'un quart de million de personnes. The Daily Star, le 6 août 1934, 1. 
par de Maisonneuve s'ouvrent officiellement à Montréal. Présidées par le maire Raynault et par l'archevêque de Montréal, Joseph Charbonneau, ces cérémonies sont relativement sobres ${ }^{88}$. Le $1^{\text {er }}$ janvier suivant, une nouvelle cérémonie présidée par les mêmes acteurs a lieu à la Place d'Armes. Par un temps glacial, on y dévoile une plaque de bronze au pied du monument à Maisonneuve. Comme la plupart des manifestations mineures qui parsèment les mois de janvier et février, ces deux événements sont assortis de services religieux célébrés en l'honneur du tricentenaire.

Mai constitue le seul moment fort des fêtes montréalaises. Il commence doucement, le 3, avec de somptueuses cérémonies dédiées à la Vierge à l'église Notre-Dame-des-Victoires. Le 13, les écoles catholiques de Montréal célèbrent à leur tour, individuellement et selon des programmes variés, le tricentenaire de la fondation de la ville ${ }^{89}$. C'est toutefois les 17 et 18 mai, dates historiques de la prise de possession de l'île par les Montréalistes, que se déroulent les cérémonies les plus importantes. La première journée est dédiée aux fêtes religieuses du tricentenaire, sous l'égide du cardinal Villeneuve ${ }^{90}$ et du délégué apostolique Ildebrando Antoniutti. Une grand-messe est célébrée dans le parc du mont Royal devant plus de 50000 personnes. Dans l'après-midi, le Très Saint-Sacrement est exposé à l'adoration des quelque 300000 Montréalais venus se recueillir. En fin de journée, ce recueillement fait place à des festivités d'une autre nature. Encadrée par plusieurs corps de cadets et par l'Union Jack, la fanfare des Grenadiers Guards présente un concert entièrement composé d'œuvres canadiennes-françaises qui se termine par un feu d'artifice offert à la ville par La Presse et dont les pièces maîtresses sont les portraits illuminés de Paul Chomedey de Maisonneuve et du roi George VI ${ }^{91}$. Le 18 mai au matin, les autorités civiles, soit le maire Raynault, les maires des différentes municipalités de l'île de Montréal et les présidents d'associations montréalaises attachées aux fêtes ${ }^{92}$, se réunissent à la Place d'Armes et entonnent le "Ô Canada "

88. J.-P. Héroux, op. cit., 37-40.

89. «...chants, cantiques, musique, danse, lectures, récitations, dialogues, sketches, causeries, joutes, débats, projections, dévoilement d'images ou de bustes représentant les principaux personnages de notre histoire, tableaux vivants, bref, tout un ensemble présenté avec art et exécuté avec amour...", Héroux, op. cit., 105.

90. "Et c'est le seul "enfant" de Montréal à jusqu'ici avoir reçu l'honneur de la pourpre qui vient magnifier, de son éclat romain, nos fêtes liturgiques. », La Patrie, le 16 mai 1942, 24.

91. J.-P. Héroux, op. cit., 131.

92. J.-P. Héroux, op. cit., 145-147. 
devant le monument à de Maisonneuve. Une foule d'organismes sont représentés. Malgré la prédominance des organisations francophones, on retrouve des représentants anglophones d'instances comme l'Université McGill, le Montreal Board of Trade, la St. Patrick's Society of Montreal et le B'Nai B'rith. C'est une des seules fois où un ensemble aussi diversifié d'acteurs sera réuni. Après une courte réception à l'hôtel de ville, un cortège à cheval transporte le maire, les membres du CTCM et une soixantaine d'invités à travers la ville. Le groupe visitera six congrégations religieuses de la ville ayant joué un rôle clé dans son développement pour leur offrir ses hommages. Il s'agit des Sœurs Hospitalières de l'Hôtel-Dieu, des Sœurs de la Congrégation de Notre-Dame, des Pères Franciscains, des Sœurs Grises, des Jésuites et des Sulpiciens ${ }^{93}$. Au même moment, plus de 5000 citoyens de la ville participent, encadrés par des guides, à un grand "pèlerinage historique" qui les conduit, à travers les rues du Vieux Montréal, d'un site historique à l'autre. En fin de soirée, de nouvelles manifestations musicales ont lieu. Dans le parc du mont Royal, la fanfare des Grenadiers Guards reprend du service pour le grand public, alors que les invités d'honneur de la CTCM assistent à un concert plus classique au Forum.

Juin est dominé par la fête de la Saint-Jean-Baptiste à laquelle les célébrations du tricentenaire sont étroitement associées. On retrouve leur trace dans les thématiques évoquées par les dix-neuf chars allégoriques qui défilent le 24. Dans les dix-neuf tableaux, dont au moins quinze sont associés uniquement aux événements $d u x{ }^{e}{ }^{e}$ siècle, on retrace les grandes étapes de l'histoire de Montréal (en fait, de Ville-Marie) ${ }^{94}$. Dans les mois suivants, différentes fêtes mineures ponctuent le calendrier. En juillet, un «festival national» est l'occasion de différentes compétitions sportives et de manifestations historiques et culturelles. En août, de nouveaux hommages sont offerts à la Vierge ; puis en septembre s'ouvre une impressionnante exposition missionnaire rendant hommage tant aux efforts de l'Église dans le monde qu'aux origines de la ville ${ }^{95}$. En octobre, une nouvelle plaque commémorative est dévoilée sur le site de la fondation de l'établissement initial de Montréal, «le Berceau de Montréal ${ }^{96}$ ». Enfin, le

93. La Patrie, le 19 mai 1942, 4.

94. J.-P. Héroux, op. cit., 221-222.

95. Cette exposition missionnaire a fait l'objet de la thèse de France Lord, La muette éloquence des choses: collections et expositions missionnaires de la Compagnie de Jésus au Québec, de 1843 à 1946, thèse de Ph.D. (Histoire), Université de Montréal, 1999, 360 p.

96. J.-P. Héroux, op. cit., 254. 
13 décembre 1942, après une représentation de l'opéra-comique canadien "Le Père des Amours», le maire Raynault annonce la dissolution de la $\mathrm{CTCM}^{97}$.

\section{CONCLUSION}

Le 10 août 1934, le TCCC est officiellement dissout. Herbert H. Alley se charge de régler les nombreuses questions financières restées en suspens à la fin des cérémonies officielles. Trouvant peut-être que l'épilogue s'étire inutilement, l'administration municipale renvoie Alley et sa secrétaire le 15 octobre $^{98}$. Le 31 décembre, une courte cérémonie est organisée par le maire et le conseil de ville pour clore officiellement l'année du centenaire. Finalement, en janvier 1935, l’instigateur des célébrations, le maire Stewart, quitte la vie politique.

À Montréal, les fêtes se terminent officiellement le 13 décembre. Par rapport aux ambitions initiales des organisateurs, elles auront plus vivoté que vécu. Néanmoins, le maire Adhémar Raynault est en mesure d'affirmer:

Nos projets ambitieux d'avant guerre ont été forcément remisés, vu que les ressources financières sur lesquelles nous comptions pour les réaliser ont été réduites à leur plus simple expression et que les nécessités d'une guerre universelle ont accaparé le maximum de nos activités. Tout de même, nous croyons que les fêtes imposantes des 17 et 18 mai derniers laisseront dans l'esprit de notre peuple un souvenir ineffaçable ${ }^{99}$.

Toutefois, à Toronto comme à Montréal, les fêtes laissent derrières elles peu de traces et l'historiographie subséquente leur accordera occasionnellement quelques lignes.

En évoquant ces deux séries de célébrations, mon ambition était de mettre en évidence les principales limites de l'exercice commémoratif comme outil identitaire. À Montréal comme à Toronto, la préparation et l'exécution des fêtes se font dans un contexte peu propice à ce genre

97. «On vous a dit que la Commission du III Centenaire vient prendre congé du public de Montréal ce soir, et vous êtes en droit de vous demander pourquoi elle est si empressée de mourir, puisqu'elle ne doit expirer que le 31 décembre. Deux raisons s'imposent à cet effet. En premier lieu, elle ne veut pas attendre d'être passée à l'état de cadavre pour se présenter devant vous; [...] elle veut mourir en beauté, aux accords de la musique et dans la gaité d'une idylle franchement canadienne... » Adhémar Raynault, cité dans J.-P. Héroux, op. cit., 263.

98. Prévenu d'avance, Alley écrit au greffier de la ville pour l'informer des questions encore en suspend. CTA, RG250, série 1, boîte 4, dossier 2, lettre de Alley à Somers, le 13 octobre 1934.

99. J.-P. Héroux, op. cit., 264. 
d'activités. Dans le premier cas, les ambitions initiales des organisateurs sont limitées par le contexte économique difficile de la crise, alors que dans le deuxième, ces ambitions sont pratiquement réduites à néant par le contexte de guerre. De même, l'histoire générale des deux villes, leur trajectoire dans l'espace économique canadien, conditionne dans une certaine mesure le caractère et le ton des commémorations.

Notons que si ce contexte "large" des fêtes affecte le contenu des discours et de pratiques commémoratives observés, on note au départ plus de similarités que de différences au niveau du personnel engagé dans leur organisation et des programmes qu'ils élaborent. À Montréal comme à Toronto, les programmes de base des fêtes sont centrés sur des thématiques similaires: célébration du passé de la ville à des fins pédagogiques mais aussi commerciales et touristiques. On y propose des festivités sportives, militaires, religieuses, culturelles et patriotiques s'adressant à la majeure partie de la communauté civique. Dans les deux cas, on espère favoriser la venue de touristes, surtout américains,. Dans cette perspective, on envisage des travaux importants d'embellissement de la ville mettant à l'ouvrage les milliers de chômeurs victimes de la crise économique. On a affaire à des groupes similaires : majoritairement des membres de l'élite économique, politique et culturelle locale qui espèrent favoriser une relance dans leurs domaines respectifs.

On notera toutefois comment, dans les deux villes, les idées et l'imagerie finalement mises de l'avant - celles d'un nationalisme canadienanglais aux accents impérialistes dans le premier cas, d'un nationalisme traditionaliste canadien-français dans l'autre - sont destinés à décliner progressivement dans les deux ou trois décennies qui suivent les célébrations étudiées ici.

Enfin, faire des célébrations du centenaire de l'incorporation de Toronto ou du tricentenaire de la fondation de Montréal de simples exercices identitaires de la part d'élites essayant d’imposer leur hégémonie aux classes populaires, c'est ignorer les autres fonctions de ces fêtes. Elles représentent à la fois un divertissement spectaculaire et une occasion de relancer ou de nourrir l'économie des deux villes à travers le tourisme. De plus, même si plusieurs composantes des fêtes sont fermées aux classes populaires, ils sont des dizaines - parfois des centaines - de milliers à participer aux festivités. Tout au long des fêtes torontoises et montréalaises, les considérations idéologiques et identitaires n’entrent pas tant en concurrence les unes avec les autres qu’avec les impératifs matériels et économiques liés à l'organisation de telles célébrations. 